\title{
Phase transitions in Proof Theory
}

\author{
Lev Gordeev ${ }^{1}$ and Andreas Weiermann ${ }^{2}$ \\ ${ }^{1}$ Tübingen University, Germany \\ ${ }^{2}$ Ghent University, Belgium
}

\begin{abstract}
Using standard methods of analytic combinatorics we elaborate critical points (thresholds) of phase transitions from provability to unprovability of arithmetical well-partial-ordering assertions in several familiar theories occurring in the reverse mathematics program.
\end{abstract}

Keywords: phase transitions, proof theory, reverse mathematics, asymptotics, analytic combinatorics, well partial orderings, Higman-Kruskal-Friedman theorems

\section{Introduction}

Consider a familiar (presumably consistent) arithmetical theory $T$ and a sufficiently complicated arithmetical assertion $A(r)$ with computable real parameter $r>0$. Let us assume that $A(r)$ is true, $T$ provable for small values of $r, T$-unprovable for large values of $r$, and that $A$ is monotone with respect to $T$-unprovability. We are interested in classifying exactly the threshold point $t$ at which the phase transition from $T$-provability to $T$-unprovability happens. That is, we wish to find $t$ such that $A(r)$ is provable (unprovable) in $T$ for $r<t(r>t)$.

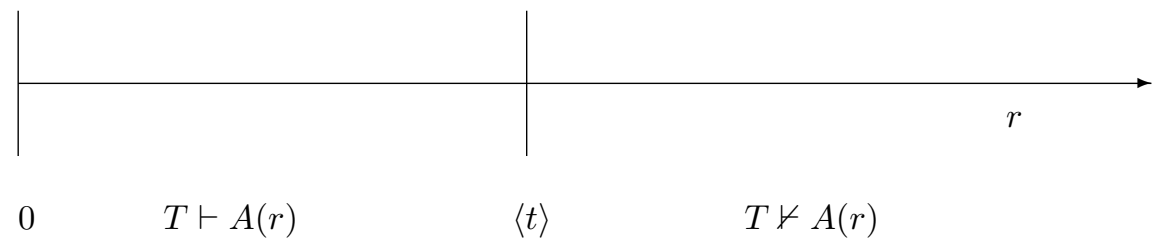

If $A$ is a mathematically interesting assertion such a result will provide valuable general insight into Gödel incompleteness and the more special into the mathematical $T$-incompleteness. Gödels first incompleteness result from 1931 states that there are true assertions about the natural numbers which do not follow from the Peano axioms. Since 1931 many researchers have been looking for natural examples of such assertions and breakthroughs have been obtained in the seventies-eighties by Paris (along with Harrington and Kirby[14, 11]) and Friedman [16] who produced mathematically interesting independence results in Ramsey theory and well-order and well-quasi-order theory, thus showing definitely that Gödel's incompleteness matters to mathematics. The phase transition program looks for a fresh contribution here. Quite interestingly in all situations which have been analyzed non logical techniques, like 
for example Tauberian theory, analytic combinatorics and even the theory of the Riemann zeta function and L-function theory, played an intrinsic role, whereas on the logical side proof theory and the theory of subrecursive hierarchies have been used to a great extent [2, 17, 18, 20, 21]. We continue this program by investigating $T$-(un)provability of assertions regarding well partial-orderedness of sets of noniterated and iterated finite sequences and trees, where $T$ is not restricted to Peano Arithmetic, while ranging over subsystems of second-order arithmetic featuring in Friedman-Simpson's reverse mathematics program [5, 6, 15]; these theories can be characterized (formalized) by familiar principles (theorems) of the ordinary mathematics, rather than abstract set theory only. The required thresholds are obtained from analytic combinatorics using methods of Flajolet and Sedgewick [4]. The possibility to calculate precise thresholds for the proof theoretic phase transitions in question is a newly discovered (by the second author) phenomenon that brings together the methods of analytic combinatorics and proof theoretic ordinal analysis (including well-partial-orderedness of nested finite sequences and trees elaborated by the first author). Analytic combinatorics enables to determine the upper bound of $T$-provability, and, on the hand, to refine Friedman's finite miniaturizations (via the König lemma) of the well partial-orderedness, which yields the corresponding lower bound of $T$-unprovability. As a whole this provides a precise characterization of $T$-(un)provability in purely mathematical terms. It is still a mystery why these two entirely different concepts provide the same, respectivey upper and lower, bounds, i.e. the desired threshold $t$. Further investigations with regard to the formal systems $T$ of higher proof theoretic strength may clarify the background. The results presented in the paper provide a useful and highly nontrivial applications of classical analytic methods (e.g., the enumeration of certain combinatorial classes) to traditional "discrete" proof theory and the reverse mathematics categorizing the strengths of classical logical frameworks.

\section{Basic definitions and results}

\subsection{Theories $T$}

Our basic arithmetical theories include Peano Arithmetic, PA, and second-order arithmetical theories from the reverse mathematics program, whose logic designations are $A C A_{0}, \Pi_{1}^{0} C A_{0}, \Delta_{1}^{1} C A, A T R_{0}, \Pi_{1}^{1} C A_{0}$, $\Delta_{2}^{1} \mathrm{CA}, \Pi_{1}^{1} \mathrm{TR}_{0}$, and their iterated extensions $\Pi_{1}^{0} \mathrm{CA}_{<\lambda}, \Pi_{1}^{1} \mathrm{CA}_{<\lambda}$ and $\Pi_{1}^{1} \mathrm{CA}_{0}^{(d)}, d>0$, for $\lambda$ ranging over primitive recursive limit ordinals. Recall that second order arithmetic is a formal theory of the natural numbers and sets of natural numbers. Abbreviations "CA" and "TR" stand for "comprehension axiom" and "transfinite recursion", respectively, whose expressive power is determined by the corresponding prefix " $A$ ", " $\Pi_{1}^{0}$ ", " $\Delta_{1}^{1}$ ", " $\Pi_{1}^{1}$ " or " $\Delta_{2}^{1}$ "; subscript " " means that only set-restricted axiom of ordinary induction is allowed. To put it more exactly, $\Phi$-comprehension $\Phi C A$ allows to form the set of natural numbers satisfying formula $\Phi$, i.e. $\{x: \Phi(x)\}$, while A refers to arbitrary arithmetical formula (one with no bound set variables, although possibly containing set parameters). Thus $\mathrm{ACA}_{0}$ allows to form the set of natural numbers satisfying an arbitrary arithmetical formula. $\Phi C \mathrm{~A}_{<\lambda}$ allows to iterate $\Phi$-comprehension $\alpha$ times, for any $\alpha<\lambda$, and $\Phi$ TR states that $\Phi$-comprehension functional can be iterated transfinitely along any well ordering starting with any set. Furthermore $\Pi_{1}^{0}\left(\Pi_{1}^{1}\right)$ refers to formulas $\Phi$ having (in prenex form) only universal unbounded numerical quantifiers $\forall x$ (one universal set quantifier $\forall X$ and arbitrary numerical quantifiers) and by the same token $\Delta_{1}^{1}\left(\Delta_{2}^{1}\right)$ refers to $\Phi$ admitting both $\forall X$ and $\exists X(\forall X \exists Y$ and $\exists X \forall Y)$ set quantifier prenex forms. Summing up $\Pi_{1}^{i} C \mathrm{~A}_{0}(i=0,1)$ adds to $A C A_{0}$ the comprehension axiom (scheme) for $\Pi_{1}^{i}$ formulas, $\Delta_{i}^{1} \mathrm{CA}(i=1,2)$ adds to $\mathrm{ACA}_{0}$ the comprehension axiom (scheme) for $\Delta_{i}^{1}$ formulas, and general scheme of ordinary induction, $\mathrm{ATR}_{0}\left(\Pi_{1}^{1} \mathrm{TR}_{0}\right)$ adds to $\mathrm{ACA}_{0}$ an axiom 
stating that any arithmetical $\left(\Pi_{1}^{1}\right)$ functional can be iterated transfinitely along any well ordering starting with any set, $\Pi_{1}^{i} C \mathrm{~A}_{<\lambda}(i=0,1)$ adds to $\mathrm{ACA}_{0}$ an axiom stating that any $\Pi_{1}^{i}$ functional can be iterated transfinitely along any $\alpha<\lambda$. Now $\Pi_{1}^{1} \mathrm{CA}_{0}^{(d)}$ is defined by recursion on $d>0$ by $\Pi_{1}^{1} \mathrm{CA}_{0}^{(1)}:=\Pi_{1}^{1} \mathrm{CA}_{0}$, $\Pi_{1}^{1} \mathrm{CA}_{0}^{(d+1)}:=\Pi_{1}^{1} \mathrm{CA}<o\left(\Pi_{1}^{1} \mathrm{CA}_{0}^{(d)}\right)$, where $o(T)$ is the canonical proof theoretic (primitive recursive) ordinal of $T$. Note that an arbitrary arithmetical formula $F$ is provable in $T$ iff it is provable in $\mathrm{ACA}_{0}$ extended by the transfinite induction scheme along any $\alpha<o(T)$. The following connections are well-known, where $\varphi^{(d)}(0)$ are strictly increasing ordinals defined by $\varphi^{(0)}(0):=\varphi_{0}(0)=1, \varphi^{(1)}(0):=\varphi_{1}(0)=$ $\varepsilon_{0}, \cdots, \varphi^{(d+1)}(0):=\varphi_{\varphi^{(d)}(0)}(0)$, and $\Gamma_{0}:=\sup _{d<\omega} \varphi^{(d)}(0)$, where $\varphi_{\alpha}(\beta)$ are Veblen predicative ordinals such that for any $\alpha, \beta, \gamma: \varphi_{0}(\beta)=\omega^{\beta}, \varphi_{1}(\beta)=\varepsilon_{\beta}$ and $\alpha<\beta \Rightarrow \varphi_{\alpha}\left(\varphi_{\beta}(\gamma)\right)=\varphi_{\beta}(\gamma)$.

1. $\mathrm{PA}$ and $\mathrm{ACA}_{0}$ prove the same arithmetical sentences and $o(\mathrm{PA})=o\left(\mathrm{ACA}_{0}\right)=\varepsilon_{0}, \Delta_{1}^{1} \mathrm{CA}=$ $\Pi_{1}^{0} \mathrm{CA}_{<\varepsilon_{0}}$ and $o\left(\Delta_{1}^{1} \mathrm{CA}\right)=\varphi^{(2)}(0), o\left(\Pi_{1}^{0} \mathrm{CA}_{<\varphi^{(d)}(0)}\right)=\varphi^{(d+1)}(0), \mathrm{ATR}_{0}=\Pi_{1}^{0} \mathrm{TR}_{0}=$ $\bigcup_{d<\omega} \Pi_{1}^{0} \mathrm{CA}_{<\varphi^{(d)}(0)}$ and $o\left(\mathrm{ATR}_{0}\right)=\Gamma_{0}$.

2. $\Delta_{2}^{1} \mathrm{CA}=\Pi_{1}^{1} \mathrm{CA} \mathrm{\varepsilon}_{0}, \Pi_{1}^{1} \mathrm{TR}_{0}=\bigcup_{d<\omega} \Pi_{1}^{1} \mathrm{CA}_{0}^{(d)}$. Moreover $\Gamma_{0}<o\left(\Pi_{1}^{1} \mathrm{CA}_{0}\right)<o\left(\Delta_{2}^{1} \mathrm{CA}\right)<$ $o\left(\Pi_{1}^{1} \mathrm{TR}_{0}\right)$ (these theories require more specific ordinal notations).

Recall [8, 9] that the following generalizations of the Kruskal-Friedman tree theorems are equivalent to our strongest theory $\Pi_{1}^{1} T R_{0}$ over $A C A_{0}$; consequently, these theorems provide the reverse-mathematics characterizations of $\Pi_{1}^{1} T R_{0}$.

- The set of finite ordered trees whose vertices are labelled by countable ordinals is well-partialordered by homeomorphic embeddability with the symmetric gap-condition ([8], see also Appendix 2 below).

- The set of finite ordered trees whose edges are labelled by countable ordinals is well-partial-ordered by homeomorphic embeddability such that every edge is mapped onto a path with greater-or-equal labels (see [12] for proof in stronger theory $\Pi_{2}^{1} \mathrm{CA}{ }^{(i)}$.

\subsection{Assertions $A(r)$}

We consider the following combinatorial classes $C$, where $d \geq 1$, while $\operatorname{Se}[X]$ and $\operatorname{Tr}[X]$ denote the finite nonempty sequences and planted plane-trees (also called ordered trees), respectively, with labels from $X$ (see more precise descriptions in Appendix 1 below).

1. $\mathrm{S}^{d}$, where $\mathrm{S}^{1}:=\mathbb{N}, \mathrm{S}^{d+1}:=\mathrm{Se}\left[\mathrm{S}^{d}\right]$.

2. $\operatorname{Ts}^{d}$, where $\operatorname{Ts}^{d}:=\operatorname{Tr}\left[\mathrm{S}^{d}\right]$.

3. $\mathrm{TT}^{d}$, where $\mathrm{TT}^{1}:=\mathrm{Ts}^{1}, \mathrm{TT}^{d+1}:=\operatorname{Tr}\left[\mathrm{TT}^{d}\right]$.

(i) $\Pi_{2}^{1}$ formulas have set quantifier prenexes $\forall X \exists Y$. 
Every class $C$ under consideration is supplied with natural additive weight function $|\cdot|: C \rightarrow \mathbb{N}_{+}$and well-partial-ordering $\unlhd_{C}$ (or just $\unlhd$ ) being the corresponding iterated homeomorphic embedding with the symmetric gap condition [16, 7, 8, 9] (see also Appendix 1). For any $r \in \mathbb{R}_{+}$, we let (ii)

$$
A(r):=\operatorname{SWP}\left(C, r^{-1}\right)
$$

where for any $a \in \mathbb{R}_{+}$, SWP $(C, a)$ is an abbreviation of the following slow well-partial-ordering assertion

$$
\begin{aligned}
& \left(\forall K \in \mathbb{N}_{+}\right)\left(\exists M \in \mathbb{N}_{+}\right)\left(\forall x_{0}, \cdots, x_{M} \in C\right) \\
& \left((\forall i \leq M)\left(\left|x_{i}\right|<K+\log _{a}(i+1)\right) \rightarrow(\exists i<j \leq M)\left(x_{i} \unlhd x_{j}\right)\right)
\end{aligned}
$$

Note that SWP $(C, a)$ is a natural finite refinement (also called finite miniaturization) of the ordinary well-partial-ordering condition WPO $(C)$ :

$$
\left(\forall x_{0}, \cdots, x_{m}, \cdots \in C\right)(\exists i<j)\left(x_{i} \unlhd x_{j}\right)
$$

with regard to $\log _{a}(\cdot)$-weight-growing infinite sequences $\left\{x_{m}\right\}$ from $C$. That is, WPO $(C)$ says that $C$ admits no infinite $\unlhd$-bad sequence $\left\{x_{m}\right\}$, whereas SWP $(C, a)$ says that any $\log _{a}(\cdot)$-weight-growing $\unlhd$ bad sequence $\left\{x_{m}\right\}$ terminates, uniformly in $\left|x_{0}\right|$. Since WPO $(C)$ holds true, so is SWP $(C, a)$, for every $a \in \mathbb{R}_{+}$. Also note that for a given theory $T$ (as above), proof theoretic ordinal $o(T)$ (more precisely, appropriate primitive recursive well-ordering $\prec_{T}$ ) can be replaced [7, 8, 9] by the corresponding more transparent well-partial-ordering $\unlhd_{C}$ from the above list ${ }^{\text {(iii) }}$ In particular this shows that WPO $(C)$ is not provable in $T$ even for primitive recursive sequences $\left\{x_{m}\right\}$, which by standard methods implies that e.g. linear finite miniaturization

$$
\begin{aligned}
& \left(\forall K \in \mathbb{N}_{+}\right)\left(\exists M \in \mathbb{N}_{+}\right)\left(\forall x_{0}, \cdots, x_{M} \in C\right) \\
& \left((\forall i \leq M)\left(\left|x_{i}\right|<K+i\right) \rightarrow(\exists i<j \leq M)\left(x_{i} \unlhd x_{j}\right)\right)
\end{aligned}
$$

is not provable in $T$, either. On the other hand, constant finite miniaturization

$$
\begin{aligned}
& \left(\forall K \in \mathbb{N}_{+}\right)\left(\exists M \in \mathbb{N}_{+}\right)\left(\forall x_{0}, \cdots, x_{M} \in C\right) \\
& \left((\forall i \leq M)\left(\left|x_{i}\right|<K\right) \rightarrow(\exists i<j \leq M)\left(x_{i} \unlhd x_{j}\right)\right)
\end{aligned}
$$

is trivially provable in $T$. So the question arises for which monotone increasing real-valued functions $f$, general finite miniaturization

$$
\begin{aligned}
& \left(\forall K \in \mathbb{N}_{+}\right)\left(\exists M \in \mathbb{N}_{+}\right)\left(\forall x_{0}, \cdots, x_{M} \in C\right) \\
& \left((\forall i \leq M)\left(\left|x_{i}\right|<K+f(i)\right) \rightarrow(\exists i<j \leq M)\left(x_{i} \unlhd x_{j}\right)\right)
\end{aligned}
$$

is (un)provable in $T$. In particular we address this question with regard to the logarithmic functions

$$
f_{r}(i):=\log _{r^{-1}}(i+1), r \in \mathbb{R}_{+}
$$

\footnotetext{
(ii) In the sequel we denote by $\mathbb{N}_{+}$and $\mathbb{R}_{+}$the sets of positive natural numbers and computable real numbers, respectively.

(iii) Without loss of the results of this paper we can just as well replace vertex-labeled trees by the corresponding edge-labeled trees from [12].
} 
and functional thresholds $f_{t}, t \in \mathbb{R}_{+}$, characterizing the corresponding functional phase transitions from $T$-provability to $T$-unprovability. In the case $T=$ PA we have natural well-partial-ordered classes $C$ which admit functional thresholds $f_{t}$ for the threshold points $t=\frac{1}{4}, \frac{1}{2}$ and $\frac{1}{c}$, where $c$ is Otter's tree constant $c \approx 2.95576$ (see [19, 20]). In this paper we consider stronger theories $T$ and find the corresponding well-partial-ordered classes $C$ (see above) and their threshold points $t$ (see below). Obviously these $t$ are the threshold points with respect to $T, C$ and $A(r)$ under consideration. Moreover $t^{-1}$ turn out to coincide with the exponential growth rates of the canonical enumerations of $C$.

\subsection{Phase transition results}

Theorem 1 For any $r \in \mathbb{R}_{+}$, basic threshold conditions:

1. $r<t \Rightarrow T \vdash \operatorname{SWP}\left(C, r^{-1}\right)$,

2. $r>t \Rightarrow T \nvdash \operatorname{SWP}\left(C, r^{-1}\right)$,

hold true for any triple $\langle T, C, t\rangle$ from the following tableau, where $d>1$.

\begin{tabular}{|c|c|c|}
\hline$T$ & $C$ & $t$ \\
\hline PA & $\mathrm{S}^{2}$ & $\frac{1}{2}$ \\
\hline$\Delta_{1}^{1} \mathrm{CA}$ & $\mathrm{S}^{3}$ & $\frac{1}{3}$ \\
\hline$\Pi_{1}^{0} \mathrm{CA}_{<\varphi^{(d)}(0)}$ & $\mathrm{S}^{d+1}$ & $\frac{1}{d+1}$ \\
\hline$\Pi_{1}^{1} \mathrm{CA}_{0}$ & $\mathrm{Ts}^{1}=\mathrm{TT}^{1}$ & $\frac{1}{8}(\sqrt{17}-1) \approx 0.3903882032$ \\
\hline$\Delta_{2}^{1} \mathrm{CA}$ & $\mathrm{Ts}^{2}$ & $\frac{1}{4}(\sqrt{5}-1) \approx 0.3090169945$ \\
\hline$\Pi_{1}^{1} \mathrm{CA}_{<\varphi^{(d)}(0)}$ & $\mathrm{Ts}^{d}$ & $\frac{1}{8}\left(\sqrt{d^{2}+16}-d\right)$ \\
\hline$\Pi_{1}^{1} \mathrm{CA}_{0}^{(d)}$ & $\mathrm{TT}^{d}$ & $\frac{1}{8}(\sqrt{17}-1)$ \\
\hline
\end{tabular}

Corollary 2 Since $\mathrm{ATR}_{0}=\bigcup_{d<\omega} \Pi_{1}^{0} \mathrm{CA}_{<\varphi^{(d)}(0)}$ and $\Pi_{1}^{1} \mathrm{TR}_{0}=\bigcup_{d<\omega} \Pi_{1}^{1} \mathrm{CA}_{0}^{(d)}$, the theorem yields the corresponding limit phase transition conditions:

$I^{\forall} . r<t_{d} \Rightarrow T \vdash(\forall d) \operatorname{SWP}\left(C_{d}, r^{-1}\right)$,

$2^{\forall} . r>t_{d} \Rightarrow T \nvdash(\forall d) \operatorname{SWP}\left(C_{d}, r^{-1}\right)$,

for any triple $\left\langle T, C_{d}, t_{d}\right\rangle$ from the following tableau, $d>0$.

\begin{tabular}{llll|}
\hline$T$ & & $C_{d}$ & $t_{d}$ \\
\cline { 1 - 1 } $\mathrm{ATR}_{0}$ & & $\mathrm{~S}^{d}$ & $\frac{1}{d}$ \\
$\Pi_{1}^{1} \mathrm{TR}_{0}$ & & $\mathrm{TT}^{d}$ & $\frac{1}{8}(\sqrt{17}-1) \approx 0.3903882032$ \\
\hline
\end{tabular}


Conjecture 3 For any $r \in \mathbb{R}_{+}$and $\langle T, C, t\rangle$ as in the theorem, the conclusion can be sharpened to:

$1^{+} . r \leq t \Rightarrow \mathrm{PA} \vdash \operatorname{SWP}\left(C, r^{-1}\right)$,

2. $r>t \Rightarrow T \nvdash \operatorname{SWP}\left(C, r^{-1}\right)$,

i.e., in graphic form

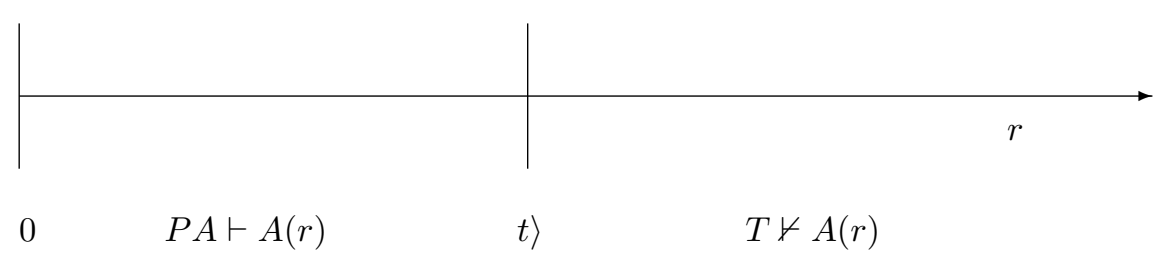

Subsequently we prove the desired sharp threshold condition $1^{+}$for

$$
T=\mathrm{PA}, C=\mathrm{S}^{2}, t=\frac{1}{2}
$$

and suppose that the whole conjecture is provable analogously. However, in the general case, the details of calculation seem to be rather involved.

\section{Methods}

Proofs of our results use two entirely different methods - analytic combinatorics and proof-theoretic ordinal analysis. The former method deals with $T$-provability and the latter with $T$-unprovability. For purely technical reasons we replace $\operatorname{SWP}(C, a)$ by $\operatorname{SWP}(C, R, \delta)$ :

$$
\begin{aligned}
& (\forall K \in \mathbb{N})(\exists M \in \mathbb{N})\left(\forall x_{0}, \cdots, x_{M} \in C\right) \\
& \left((\forall i \leq M)\left(\left|x_{i}\right| \leq K+\delta \cdot\left\lceil\log _{R}(i+1)\right\rceil\right) \rightarrow(\exists i<j \leq M)\left(x_{i} \unlhd x_{j}\right)\right)
\end{aligned}
$$

$\left(R, \delta \in \mathbb{R}_{+}\right)$and observe that our threshold conditions $1,1^{+}$and 2 are equivalent to the following $l_{t}, l_{t}^{+}$and $2_{t}$, respectively:

$$
\begin{aligned}
& 1_{t} . \delta<1 \Rightarrow T \vdash \operatorname{SWP}\left(C, t^{-1}, \delta\right), \\
& 1_{t}^{+} . \delta \leq 1 \Rightarrow \mathrm{PA} \vdash \operatorname{SWP}\left(C, t^{-1}, \delta\right), \\
& 2_{t} . \delta>1 \Rightarrow T \nvdash \operatorname{SWP}\left(C, t^{-1}, \delta\right) .
\end{aligned}
$$

\subsection{T-provability}

\subsubsection{Threshold condition 1}

We prove condition $l_{t}$ (see above). In the sequel we let $R:=t^{-1}$. Suppose $\delta<1$. To conclude that SWP $(C, R, \delta)$ is provable in $T$ (actually in PA), it will suffice to prove that for any $K$ and sufficiently large $M$ we have 


$$
\#\left\{x \in C:|x| \leq K+\delta \cdot\left\lceil\log _{R}(M+1)\right\rceil\right\} \leq M
$$

which implies that for any $x_{0}, \cdots, x_{M} \in C:\left|x_{i}\right| \leq K+\delta \cdot\left\lceil\log _{R}(i+1)\right\rceil$ there are $i<j$ such that $x_{i}=x_{j}$ and hence $x_{i} \unlhd x_{j}$, as required. Now $(*)$ follows by standard counting arguments via the ordinary generating functions (OGF) $G(z)$ and $G_{\leq}(z)=(1-z)^{-1} G(z)$ satisfying

$$
\begin{aligned}
{\left[z^{n}\right] G(z) } & =\#\{x \in C:|x|=n\} \\
{\left[z^{n}\right] G_{\leq}(z) } & =\#\{x \in C:|x| \leq n\}
\end{aligned}
$$

where $\left[z^{n}\right] f(z)$ denotes the coefficient of $z^{n}$ in the Taylor power series expansion of $f(z)$ at $z=0$ (cf. [4I). Actually it will suffice to verify that exponential order of $\left[z^{n}\right] G(z)$ coincides with chosen parameter $R$, i.e. $\left[z^{n}\right] G(z) \bowtie R^{n}=t^{-n}$. To this end we determine OGF $G(z)$ for every $C$ under consideration $(d>0)$ :

\begin{tabular}{|ll|}
\hline$C$ & $\frac{G(z)=G_{C}(z)}{\frac{z}{1-d z}}$ \\
$\mathrm{~S}^{d}$ & $\frac{1}{2}\left(1-\sqrt{1-\frac{4 z^{2}}{1-d z}}\right)$ \\
$\mathrm{Ts}^{d}$ & $\frac{1}{2}\left(1-\sqrt{1-\frac{4 z^{2}}{1-z}}\right)$ \\
$\mathrm{TT}^{1}=\mathrm{Ts}^{1}$ & $\frac{1}{2}\left(1-\sqrt{1-2 z\left(1-\sqrt{1-\frac{4 z^{2}}{1-z}}\right)}\right)$ \\
$\mathrm{TT}^{2}$ & $G_{\mathrm{TT}^{d+1}}(z)=\frac{1}{2}\left(1-\sqrt{1-4 z G_{\mathrm{TT}^{d}}(z)}\right)$ \\
$\mathrm{TT}^{d+1}$ &
\end{tabular}

and calculate its dominant singularity $\rho$, which yields the required exponential order $R=\rho^{-1}$ :

\begin{tabular}{llll}
\multicolumn{1}{l}{$\frac{\rho}{\mathrm{S}^{d}}$} & & $R=t^{-1}$ \\
$\mathrm{Ts}^{-1}$ & $\frac{1}{8}\left(\sqrt{d^{2}+16}-d\right)$ & $\frac{1}{2}\left(\sqrt{d^{2}+16}+d\right)$ \\
$\mathrm{TT}^{1}=\mathrm{Ts}^{1}$ & $\frac{1}{8}(\sqrt{17}-1)$ & $\frac{1}{2}(\sqrt{17}+1) \approx 2.561552813$ \\
$\mathrm{TT}^{2}$ & $\frac{1}{8}(\sqrt{17}-1)$ & $\frac{1}{2}(\sqrt{17}+1)$ \\
$\mathrm{TT}^{d+1}$ & $\rho_{\mathrm{TT}^{d+1}}=\rho_{\mathrm{TT}^{d}}$ & $\frac{1}{2}(\sqrt{17}+1)$
\end{tabular}

From $\left[z^{n}\right] G(z) \bowtie R^{n}$ we easily obtain $\left[z^{n}\right] G_{\leq}(z) \bowtie R^{n}$, which yields an upper bound estimate of $\left[z^{n}\right] G_{\leq}(z)=\#\{x \in C:|x| \leq n\}:$

$$
(\forall \epsilon>0)\left(\exists N \in \mathbb{N}_{+}\right)(\forall n \geq N)\left(\left[z^{n}\right] G_{\leq}(z)<(R+\epsilon)^{n}\right)
$$

To complete the proof of $(*)$ we argue by contraposition. 
Suppose $(\forall L>0)(\exists M \geq L)\left(M<\left[z^{K+\delta \cdot\left\lceil\log _{R}(M+1)\right\rceil}\right] G_{\leq}(z)\right)$ and let $\epsilon:=\frac{1}{2} R \ln (R)\left(\delta^{-1}-1\right)$. For sufficiently large $M$ this yields

$$
\begin{aligned}
M & <\left[z^{\left.K+\delta \cdot\left[\log _{R}(M+1)\right\rceil\right]} G_{\leq}(z)\right. \\
& <(R+\epsilon)^{K+\delta} \cdot(R+\epsilon)^{\delta \cdot \log _{R}(M)}=(R+\epsilon)^{K+\delta} \cdot M^{\delta \frac{\ln (R+\epsilon)}{\ln (R)}} \\
& <(R+\epsilon)^{K+\delta} \cdot M^{\delta\left(1+\frac{\epsilon}{R \ln (R)}\right)}<R^{K+1} \cdot\left(1+\frac{\ln R}{2 \delta}\right)^{K+1} \cdot M^{\frac{1}{2}(\delta+1)}
\end{aligned}
$$

and hence $(\forall L>0)(\exists M \geq L)\left(M^{\frac{1}{2}(1-\delta)}<R^{K+1} \cdot\left(1+\frac{\ln R}{2 r}\right)^{K+1}\right)$, which is impossible by $1-\delta>0$. So $(\exists L>0)(\forall M \geq L)\left(M>\left[z^{K+\delta \cdot\left\lceil\log _{R}(M+1)\right\rceil}\right] G_{\leq}(z)\right)$, Q.E.D.

\subsubsection{Sharp threshold condition $1^{+}$}

We prove condition $I_{t}^{+}$(see above) in the simplest case $C:=\mathrm{S}^{2}$ and $t=\frac{1}{2}$. Recall that $R=t^{-1}$. It will suffice to let $\delta:=1$ and show that $\operatorname{SWP}\left(\mathrm{S}^{2}, 2,1\right)$ is still provable in PA. We have $\left\langle\mathrm{S}^{2}, \unlhd\right\rangle=\left\langle\left(\mathbb{N}_{+}\right)^{*}, \unlhd\right\rangle$ where

$$
\begin{aligned}
\left\langle s_{0}, \cdots, s_{m}\right\rangle \unlhd & \left\langle s_{0}^{\prime}, \cdots, s_{m^{\prime}}^{\prime}\right\rangle \Leftrightarrow\left(\exists i_{0}, \cdots, i_{m} \leq m^{\prime}\right)(\forall j \leq m)\left(s_{j} \leq s_{i_{j}}^{\prime}\right) \\
& \wedge(\forall j<m)\left(\forall i_{j}<k<i_{j+1}\right)\left(\min \left\{s_{j}, s_{j+1}\right\} \leq s_{k}^{\prime}\right)
\end{aligned}
$$

So SWP $\left(\mathrm{S}^{2}, 2,1\right)$ reads

$$
\begin{aligned}
& \left(\forall K \in \mathbb{N}_{+}\right)\left(\exists M \in \mathbb{N}_{+}\right)\left(\forall x_{0}, \cdots, x_{M} \in\left(\mathbb{N}_{+}\right)^{*}\right) \\
& \left((\forall i \leq M)\left(\left|x_{i}\right| \leq K+\left\lceil\log _{2}(i+1)\right\rceil\right) \rightarrow(\exists i<j \leq M)\left(x_{i} \unlhd x_{j}\right)\right)
\end{aligned}
$$

Assume that $K$ is sufficiently large. Arguing by contraposition let $M:=2^{2^{K^{2}}}-1$ and suppose that $x_{0}, \cdots, x_{M} \in\left(\mathbb{N}_{+}\right)^{\star}$ is $\unlhd$-bad, i.e. $(\forall i<j \leq M) \neg\left(x_{i} \unlhd x_{j}\right)$, so in particular $(\forall i<j \leq M)\left(x_{i} \neq x_{j}\right)$. Let $[K]=\underbrace{\langle K, \cdots, K\rangle}_{K \text { times }} \in\left(\mathbb{N}_{+}\right)^{*}$.

Since $\left|x_{0}\right| \leq K, \neg\left(x_{0} \unlhd x\right)$ implies $\neg([K] \unlhd x)$, which yields $\forall i=1, \cdots, M$ :

$$
x_{i} \in\left\{\begin{array}{c}
x \in\left(\mathbb{N}_{+}\right)^{\star}: \neg([K] \unlhd x) \wedge|x| \leq K+\left\lceil\log _{2}(M+1)\right\rceil \\
=K+2^{K^{2}}
\end{array}\right\}
$$

and thereby

$$
\#\left\{x \in\left(\mathbb{N}_{+}\right)^{\star}: \neg([K] \unlhd x) \wedge|x| \leq K+2^{K^{2}}\right\} \geq M=2^{2^{K^{2}}}-1 \quad(* *)
$$

To refute $(* *)$ we estimate upper bounds of $\#\left\{x \in\left(\mathbb{N}_{+}\right)^{\star}: \neg([K] \unlhd x) \wedge|x| \leq n\right\}$. Consider the corresponding classes

$$
\mathcal{H}_{K}:=\left\{x \in\left(\mathbb{N}_{+}\right)^{\star}: \neg([K] \unlhd x)\right\}
$$

and define OGFs $H_{K}(z), H_{K, \leq}(z)$ with $\left[z^{n}\right] H_{K}(z)=\#\left\{x \in \mathcal{H}_{K}:|x|=n\right\}$ and $\left[z^{n}\right] H_{K, \leq}(z)=$ $\#\left\{x \in \mathcal{H}_{K}:|x| \leq n\right\}$. To this end consider any $x=\left\langle s_{0}, \cdots, s_{\ell}\right\rangle \in\left(\mathbb{N}_{+}\right)^{*}$ and observe that $\neg([\bar{K}] \unlhd x)$ iff any block $X \subseteq x$ consisting of successive occurrences $s_{p}, \cdots, s_{q} \geq K$, in $x$, has the length $q-p<K$. 
Thus $x$ is a chain of such $X$ having arbitrary blocks $Y=s_{q}, \cdots, s_{r}<K$ between them, $x=X$ and $x=Y$ being two special cases. This yields $\mathcal{H}_{K} \cong$

$$
\mathbb{N}_{<K}^{<\infty} \cup \mathbb{N}_{\geq K}^{<K} \cup 2 \cdot \bigcup_{i<\infty}\left(\mathbb{N}_{<K}^{<\infty} \times \mathbb{N}_{\geq K}^{<K}\right)^{i} \cup \bigcup_{i<\infty}\left(\mathbb{N}_{<K}^{<\infty} \times \mathbb{N}_{\geq K}^{<K}\right)^{i} \times\left(\mathbb{N}_{<K}^{<\infty} \cup \mathbb{N}_{\geq K}^{<K}\right)
$$

for $\mathbb{N}_{\geq K}=\left\{n \in \mathbb{N}_{+}: K \leq n\right\}, \mathbb{N}_{<K}=\left\{n \in \mathbb{N}_{+}: n<K\right\}, \mathbb{N}_{<K}^{<\infty}=\sum_{n<\infty}\left(\mathbb{N}_{<K}\right)^{n}$ and $\mathbb{N}_{\geq K}^{<K}=$ $\sum_{n=1}^{K-1}\left(\mathbb{N}_{<K}\right)^{n}$. Having this and using standard notations:

$$
\begin{aligned}
I_{\geq K}(z) & =\sum_{n=K}^{\infty} z^{n}=\frac{z^{K}}{1-z}, \quad I_{<K}(z)=\sum_{n=1}^{K-1} z^{n}=\frac{z-z^{K}}{1-z}, \\
I I_{<K}(z) & =I\left(I_{<K}(z)\right)=\frac{z-z^{K}}{1-2 z+z^{K}}, \\
I_{\geq K}^{<K}(z) & =\sum_{n=1}^{K-1}\left(I_{\geq K}(z)\right)^{n}=I_{<K}\left(I_{\geq K}(z)\right)=\frac{z^{K}(1-z)^{K-1}-z^{K^{2}}}{(1-z)^{K-1}\left(1-z-z^{K}\right)}
\end{aligned}
$$

we obtain the required OGF

$$
\begin{aligned}
H_{K}(z) & =I I_{<K}(z)+I_{\geq K}^{<K}(z)+I\left(I I_{<K}(z) \cdot I_{\geq K}^{<K}(z)\right) \cdot\left(2+I I_{<K}(z)+I_{\geq K}^{<K}(z)\right) \\
& =\frac{z(1-z)^{K}-z^{K^{2}}\left(1-z^{K}\right)}{(1-z)^{K}(1-2 z)+z^{K^{2}+1}\left(1-z^{K-1}\right)}
\end{aligned}
$$

and hence

$$
H_{K, \leq}(z)=\frac{z(1-z)^{K}-z^{K^{2}}\left(1-z^{K}\right)}{(1-z)\left((1-z)^{K}(1-2 z)+z^{K^{2}+1}\left(1-z^{K-1}\right)\right)} .
$$

Following [4] we estimate the dominant $H_{K, \leq}(z)$-pole $\rho_{K}$ :

$$
\frac{1}{2}+\left(\frac{1}{2}\right)^{K^{2}-K+3}<\rho_{K}<\frac{1}{2}+\left(\frac{1}{2}\right)^{\frac{2}{3} K^{2}-\frac{25}{21} K+\frac{5}{3}}
$$

and for sufficiently large $n$ obtain

$$
\left[z^{n}\right] H_{K, \leq}(z)<n^{K^{2}+K} \cdot\left(\frac{2}{1+\left(\frac{1}{2}\right)^{K^{2}-K+2}}\right)^{n} .
$$

For sufficiently large $K$ this yields

$$
\begin{aligned}
& \#\left\{x \in\left(\mathbb{N}_{+}\right)^{*}: \neg([K] \unlhd x) \wedge|x| \leq K+2^{K^{2}}\right\} \\
= & {\left[z^{K+2^{K^{2}}}\right] H_{K, \leq}(z)<\left(K+2^{K^{2}}\right)^{K^{2}+K} \cdot\left(\frac{2}{1+\left(\frac{1}{2}\right)^{K^{2}-K+2}}\right)^{K+2^{K^{2}}} } \\
< & 2^{2^{K^{2}}}-1=M
\end{aligned}
$$


which contradicts $(* *)$ and thereby proves SWP $\left(\mathrm{S}^{2}, 2,1\right)$ - obviously in PA, as desired.

\subsection{T-unprovability: threshold condition 2}

We prove condition $2_{t}$ (see above). Suppose $\delta>1$. We have to show that $\operatorname{SWP}(C, R, \delta)$ is not provable in $T$, where $R:=t^{-1}$. To begin with we split $C=\mathrm{S}^{d}, \mathrm{Ts}^{d}, \mathrm{TT}^{d}$ into the following subclasses $C_{k}=$ $\mathrm{S}_{k}^{d}, \mathrm{Ts}_{k}^{d}, \mathrm{TT}_{k}^{d}$, respectively $(d, k>0)$.

1. $\mathrm{S}_{k}^{1}:=\left\{n \in \mathbb{N}_{+}: n \leq k\right\}, \mathrm{S}_{k}^{d+1}:=\operatorname{Se}\left[\mathrm{S}_{k}^{d}\right]$.

2. $\operatorname{TS}_{k}^{d}:=\operatorname{Tr}\left[\mathrm{S}_{k}^{d}\right]$.

3. $\operatorname{TT}_{k}^{1}:=\mathrm{Ts}_{k}^{1}, \mathrm{TT}_{k}^{d+1}:=\mathrm{T}\left[\mathrm{TT}_{k}^{d}\right]$.

Subsequently we determine the corresponding OGF $G_{k}(z)$ with $\left[z^{n}\right] G_{k}(z)=\#\left\{x \in C_{k}:|x|=n\right\}$ :

\begin{tabular}{|c|c|}
\hline$C_{k}$ & $G_{k}(z)=G_{C_{k}}(z)$ \\
\hline $\mathrm{S}_{k}^{d}$ & $\frac{z-z^{k+1}}{1-d z+(d-1) z^{k+1}}$ \\
\hline $\mathrm{Ts}_{k}^{d}$ & $\frac{1}{2}\left(1-\sqrt{1-\frac{4 z^{2}\left(1-z^{k}\right)}{1-d z+(d-1) z^{k+1}}}\right)$ \\
\hline $\mathrm{TT}_{k}^{1}$ & $\frac{1}{2}\left(1-\sqrt{1-4 z^{2} \sum_{i=0}^{k-1} z^{i}}\right)$ \\
\hline $\mathrm{TT}_{k}^{2}$ & $\frac{1}{2}\left(1-\sqrt{1-2 z\left(1-\sqrt{1-4 z^{2} \sum_{i=0}^{k-1} z^{i}}\right)}\right.$ \\
\hline $\mathrm{TT}_{k}^{d+1}$ & $G_{\mathrm{TT}_{k}^{d+1}}(z)=\frac{1}{2}\left(1-\sqrt{1-4 z G_{\mathrm{TT}_{k}^{d}}(z)}\right)$ \\
\hline
\end{tabular}

and calculate dominant singularities $\rho_{k}$ :

\begin{tabular}{lll}
\hline$C_{k}$ & $\rho_{k}$ \\
\hline $\mathrm{S}_{k}^{d}$ & $\min \left\{y_{>0}: 1=d y-(d-1) y^{k+1}\right\}$ \\
$\operatorname{Ts}_{k}^{d}$ & $\min \left\{y_{>0}: 1=d y-(d-1) y^{k+1}+4 y^{2}\left(1-y^{k}\right)\right\}$ \\
$\operatorname{TT}_{k}^{d}$ & $\min \left\{y_{>0}: 1=4 y^{2} \sum_{i=0}^{k-1} y^{i}\right\}$ \\
\hline
\end{tabular}

This yields the corresponding exponential orders $R_{k}=\left(\rho_{k}\right)^{-1}$ of $\left[z^{n}\right] G_{k}(z)=\#\left\{x \in C_{k}:|x|=n\right\}$ and $\left[z^{n}\right] G_{k, \leq}(z)=\#\left\{x \in C_{k}:|x| \leq n\right\}$ (both), where as usual $G_{k, \leq}(z)=(1-z)^{-1} G_{k}(z)$.

Moreover $R_{k} \nearrow R$ as $k \rightarrow \infty$ where as above 


\begin{tabular}{lll|}
\hline$C$ & $R=t^{-1}$ \\
\cline { 1 - 1 } & $d$ \\
$\mathrm{TS}^{d}$ & & $\frac{1}{2}\left(\sqrt{d^{2}+16}+d\right)$ \\
$\mathrm{TT}^{d}$ & $\frac{1}{2}(\sqrt{17}+1) \approx 2.561552813$ \\
\hline
\end{tabular}

Furthermore we show that for every $C_{k}$ there exists $c_{k}>0$ such that

$$
\left(\exists N \in \mathbb{N}_{+}\right)(\forall n \geq N)\left(c_{k} \cdot\left(R_{k}\right)^{n}<\left[z^{n}\right] G_{k, \leq}(z)\right)
$$

(by Flajolet's Second Principle of Coefficient Asymptotics [4]:Thm.IV.10, p.258), which for sufficiently large $i$ yields the following lower bound estimate

$$
\begin{aligned}
& \#\left\{x \in C_{k}:|x| \leq\left\lceil\log _{R_{k}}(i+1)\right\rceil\right\} \\
= & {\left[z^{\left\lceil\log _{R_{k}}(i+1)\right\rceil}\right] G_{k, \leq}(z)>c_{k} \cdot\left(R_{k}\right)^{\log _{R_{k}}(i+1)}>c_{k} \cdot i }
\end{aligned}
$$

To complete the proof we first confirm by standard proof-theoretic arguments that linear finite miniaturization $\operatorname{SWP}_{1}(C)$ :

$$
\begin{aligned}
& \left(\forall m \in \mathbb{N}_{+}\right)(\exists k>n)\left(\forall K \in \mathbb{N}_{+}\right)\left(\exists M \in \mathbb{N}_{+}\right)\left(\forall x_{0}, \cdots, x_{M} \in C_{k}\right) \\
& \left((\forall i \leq M)\left(\left|x_{i}\right| \leq K+i\right) \rightarrow(\exists i<j \leq M)\left(x_{i} \unlhd x_{j}\right)\right)
\end{aligned}
$$

is (true but) not provable in $T$. Using the above lower bound we pass from $T$-unprovability of $\mathrm{SWP}_{1}(C)$ to $T$-unprovability of $\operatorname{SWP}_{2}(C, R)$ :

$$
\begin{aligned}
& \left(\forall m \in \mathbb{N}_{+}\right)(\exists k>n)\left(\forall K \in \mathbb{N}_{+}\right)\left(\exists M \in \mathbb{N}_{+}\right)\left(\forall x_{0}, \cdots, x_{M} \in C_{k+1}\right) \\
& \left((\forall i \leq M)\left(\left|x_{i}\right| \leq K+2\left\lceil\log _{R_{k}}(i+1)\right\rceil\right) \rightarrow(\exists i<j \leq M)\left(x_{i} \unlhd x_{j}\right)\right)
\end{aligned}
$$

(see Appendix 2 below) and by analogous arguments from $T$-unprovability of $\operatorname{SWP}_{2}(C, R)$ to the required $T$-unprovability of $\operatorname{SWP}(C, R, \delta)$ :

$$
\begin{aligned}
& (\forall K \in \mathbb{N})(\exists M \in \mathbb{N})\left(\forall x_{0}, \cdots, x_{M} \in C\right) \\
& \left((\forall i \leq M)\left(\left|x_{i}\right| \leq K+\delta \cdot\left\lceil\log _{R}(i+1)\right\rceil\right) \rightarrow(\exists i<j \leq M)\left(x_{i} \unlhd x_{j}\right)\right)
\end{aligned}
$$

also using the possibility to choose $k$ so large and $1<\delta^{\prime}<\delta$ so small that

$$
2\left\lceil\log _{R_{k}}\left(\left\lceil\log _{R_{k}}(i+1)\right\rceil+1\right)\right\rceil+\delta^{\prime} \cdot\left\lceil\log _{R_{k}}(i+1)\right\rceil \leq \delta \cdot\left\lceil\log _{R}(i+1)\right\rceil .
$$

\section{Appendix 1}

\subsection{Iterated sequences and ordered trees}

\subsubsection{Basic classes $C$}

For any set of "labels" $X$, let $\operatorname{Se}[X]:=\bigcup_{i=1}^{\infty} X^{i}$ and $\operatorname{Se}_{k}[X]:=\bigcup_{i=1}^{k} X^{i}$, where $k>0$ and $X^{i}=$ $\underbrace{X \times \cdots \times X}_{i \text { times }}$, and let $\operatorname{Tr}[X]:=\bigcup_{i=1}^{\infty} \operatorname{Tr}^{[i]}[X]$ where $\operatorname{Tr}^{[1]}[X]:=\{\bullet\} \times X, \operatorname{Tr}^{[i+1]}[X]:=\operatorname{Tr}^{[i]}[X] \cup$ 
$\{\bullet\} \times \operatorname{Se}\left[\operatorname{Tr}^{[i]}[X]\right] \times X$. Thus every node $v \in T \in \operatorname{Tr}[X]$ is assigned with the uniquely determined label $\ell(v) \in X$. We call $\mathrm{Se}[X]\left(\mathrm{Se}_{k}[X]\right)$ and $\operatorname{Tr}[X]$ the corresponding sequences (k-bounded) and labelled ordered trees, respectivelly. Moreover for any $d>0$ consider the following basic classes of iterated (or nested) sequences and ordered trees:

$\mathrm{S}^{0}:=\{\bullet\}, \mathrm{S}^{1}:=\mathrm{Se}\left[\mathrm{S}^{0}\right]=\{\bullet, \bullet \bullet, \bullet \bullet, \cdots\} \cong \mathbb{N}_{+}, \mathrm{S}^{d+1}:=\operatorname{Se}\left[\mathrm{S}^{d}\right], \mathrm{S}_{k}^{1}:=\mathrm{Se}_{k}\left[\mathrm{~S}^{0}\right]=$
$\{\underbrace{\bullet, \bullet \bullet \bullet \bullet \bullet, \cdots}_{k \text { times }}\}, \mathrm{S}_{k}^{d+1}:=\operatorname{Se}\left[\mathrm{S}_{k}^{d}\right], \mathrm{TS}^{d}:=\operatorname{Tr}\left[\mathrm{S}^{d}\right], \mathrm{TS}_{k}^{d}:=\operatorname{Tr}\left[\mathrm{S}_{k}^{d}\right], \mathrm{TT}^{1}:=\mathrm{Ts}^{1}, \mathrm{TT}^{d+1}:=$ $\operatorname{Tr}\left[\mathrm{TT}^{d}\right], \mathrm{TT}_{k}^{1}:=\mathrm{Ts}_{k}^{1}, \mathrm{TT}_{k}^{d+1}:=\operatorname{Tr}\left[\mathrm{TT}_{k}^{d}\right]$. Obviously $\mathrm{S}_{k}^{d} \subset \mathrm{S}^{d}, \mathrm{Ts}_{k}^{d} \subset \mathrm{Ts}^{d}$ and $\mathrm{TT}_{k}^{d} \subset \mathrm{TT}^{d}$. Moreover for every nested class $C$ under consideration we fix a primitive recursive enumeration $\nu_{C}: \mathbb{N} \rightarrow C$ (e.g., standard lexicographic enumeration of $C$ ), and define natural additive weight function $|\cdot|: C \rightarrow \mathbb{N}_{+}$ : each node gets weight 1 , furthermore a single node with label $x$ gets the weight $|x|+1$. (In particular each "natural number" $n \in \mathrm{S}^{1}$ gets weight $n$.) Furthermore for any $a \in C$, where $C$ of the form $\mathrm{S}^{d}, \mathrm{Ts}^{d}$ or $\mathrm{TT}^{d}$ (hence $C_{k}=\mathrm{S}_{k}^{d}, \mathrm{Ts}_{k}^{d}$ or $\mathrm{TT}_{k}^{d}$, respectively), we let $\varrho_{C}(a):=\min \left\{k: a \in C_{k}\right\}$.

\subsubsection{Basic orderings on $C$}

By $\unlhd$ and $\leq$ we denote partial and linear countable well-orderings (abbr.: wpo and wo), respectively. A wo $\mathcal{O}=\langle W, \leq\rangle$ is called a linearization of a wpo $\mathcal{W}=\langle W, \unlhd\rangle$ iff $(\forall a, b \in W)(a \unlhd b \rightarrow a \leq b)$. A wpo $\langle W, \unlhd\rangle$ is called enumerated iff it is supplied with a bijection, also called enumeration, $\nu: \mathbb{N} \rightarrow W$. For any enumerated wpo $\langle W, \unlhd, \nu\rangle$ we fix its canonical linearization $\langle W, \leq[\unlhd, \nu]\rangle$ where ${ }^{(i v)}$

$$
\begin{aligned}
a \leq & {[\unlhd, \nu] b: \Leftrightarrow(\forall i \in \mathbb{N})(\nu(i) \unlhd a \leftrightarrow \nu(i) \unlhd b) \vee } \\
& (\exists i \in \mathbb{N})(\neg(\nu(i) \unlhd a) \wedge \nu(i) \unlhd b \wedge(\forall j<i)(\nu(j) \unlhd a \leftrightarrow \nu(j) \unlhd b))
\end{aligned}
$$

Furthermore for any $C$ of the form $\mathrm{S}^{d}, \mathrm{Ts}^{d}$ or $\mathrm{TT}^{d}$ and a chosen enumeration $\nu_{C}: \mathbb{N} \rightarrow C$ (see above) we upgrade $\left\langle C, \leq\left[\unlhd, \nu_{C}\right]\right\rangle$ to $\left\langle C, \leq\left[\unlhd, \nu_{C}, \varrho_{C}\right]\right\rangle$ where

$$
a \leq\left[\unlhd, \nu_{C}, \varrho_{C}\right] b: \Leftrightarrow \varrho_{C}(a)<\varrho_{C}(b) \vee\left(\varrho_{C}(a)=\varrho_{C}(b) \wedge a \leq\left[\unlhd, \nu_{C}\right] b\right)
$$

Definition 1 Let $\leq$ be a fixed linear ordering on $X$. Given a pair of sequences $S=\left\langle s_{0}, \cdots, s_{m}\right\rangle, S^{\prime}=$ $\left\langle s_{0}^{\prime}, \cdots, s_{m^{\prime}}^{\prime}\right\rangle \in \operatorname{Se}[X]$, we say that $S$ is embeddable with the symmetric gap condition into $S^{\prime}$ (abbr: $\left.S \unlhd_{[\leq]} S^{\prime}\right)$ iff

$$
\left(\exists i_{0}, \cdots, i_{m} \leq m^{\prime}\right)(\forall j \leq m)\left(s_{j} \leq s_{i_{j}}^{\prime}\right) \wedge(\forall j<m)\left(\forall i_{j}<k<i_{j+1}\right)\left(\min \left\{s_{j}, s_{j+1}\right\} \leq s_{k}^{\prime}\right)
$$

Definition 2 Under the same assumption about $\leq$ a labelled tree $T \in \operatorname{Tr}[X]$ is homeomorphically embeddable with the symmetric gap condition into a labelled tree $T^{\prime} \in \operatorname{Tr}[X]\left(\right.$ abbr.: $\left.T \unlhd_{[\leq]} T^{\prime}\right)$ iff there is a homeomorphic embedding $h: T \rightarrow T^{\prime}$ such that $(\forall v \in T)(\ell(v) \leq \ell(h(v)))$ and $\min \left\{\ell(v), \ell\left(v^{\prime}\right)\right\} \leq$ $\ell(u)$ holds for any neighbors $v, v^{\prime}$ in any path $P \subset T$ and any $u \in h(P) \subset T^{\prime}$.

Now for any $C$ of the form $\mathrm{S}^{d}, \mathrm{Ts}^{d}$ or $\mathrm{TT}^{d}, d>0$, we determine the required wqo $\left\langle C, \unlhd_{C}\right\rangle$ and wo $\left\langle C, \leq_{C}\right\rangle$, as follows.

(iv) For any wpo $W=(W, \unlhd)$ denote by $o(\mathcal{W})$ the supremum of order types, i.e. set theoretical ordinals, of all linearizations of $W$. For all enumerated $W=(W, \nu, \unlhd)$ considered in the paper, $W_{\nu}=\left(W, \leq_{\nu}\right)$ has the order type $o(\mathcal{W})$. This conclusion fails for arbitrary enumerated wpo; in general maximal linearizations don't admit explicit arithmetical definitions (see [13]). 
1. Let $\leq_{C}:=\leq\left[\unlhd_{C}, \nu_{C}, \varrho_{C}\right]$. Note that if $C=\mathrm{S}^{1} \cong \mathbb{N}_{+}$, then $\leq_{C}$ is the canonical $\leq$ on $\mathbb{N}_{+}$.

2. If $C=\mathrm{S}^{d}$, then $\unlhd_{C}$ is defined by recursion on $d$ :

(a) If $C=\mathrm{S}^{1} \cong \mathbb{N}$, let $\unlhd_{C}:=\leq_{C}$.

(b) If $C=\mathrm{S}^{d}$ and $C^{\prime}=\mathrm{S}^{d+1}$, let $\unlhd_{C^{\prime}}:=\unlhd_{\left[\leq\left[\unlhd_{C}, \nu_{C}, \varrho_{C}\right]\right]}$ (on Se $[C]$ ).

3. If $C=\mathrm{S}^{d}$ and $C^{\prime}=\operatorname{TS}^{d}=\operatorname{Tr}\left[\mathrm{S}^{d}\right]$, let $\unlhd_{C^{\prime}}:=\unlhd_{\left[\leq_{C}\right]}$ (on $\operatorname{Tr}[C]$ ).

4. If $C=\mathrm{TT}^{d}$, then $\unlhd_{C}$ is defined by recursion on $d$ :

(a) If $C=\mathrm{TT}^{1}$, let $\unlhd_{C}:=\unlhd_{\mathrm{Ts}^{1}}$.

(b) If $C=\mathrm{TT}^{d}$ and $C^{\prime}=\mathrm{TT}^{d+1}$, let $\unlhd_{C^{\prime}}:=\unlhd_{\left[\leq\left[\unlhd_{C}, \nu_{C}, \varrho_{C}\right]\right]}$ (on $\operatorname{Tr}[C]$ ).

Note that all relations $\unlhd_{d}, \leq_{d}$ are definable in the language of PA.

\section{Appendix 2}

We let

$$
\begin{aligned}
\operatorname{SWP}_{1}^{0}(C): \quad & \left(\forall K \in \mathbb{N}_{+}\right)\left(\exists M \in \mathbb{N}_{+}\right)\left(\forall x_{0}, \cdots, x_{M} \in C\right) \\
& \left((\forall i \leq M)\left(\left|x_{i}\right| \leq K+i\right) \rightarrow(\exists i<j \leq M)\left(x_{i} \unlhd x_{j}\right)\right), \\
\operatorname{SWP}_{2}^{0}(C, R): \quad & \left(\forall K \in \mathbb{N}_{+}\right)\left(\exists M \in \mathbb{N}_{+}\right)\left(\forall x_{0}, \cdots, x_{M} \in C\right) \\
& \left((\forall i \leq M)\left(\left|x_{i}\right| \leq K+2|i|_{R}\right) \rightarrow(\exists i<j \leq M)\left(x_{i} \unlhd x_{j}\right)\right) .
\end{aligned}
$$

where $|i|_{R}:=\left\lceil\log _{R}(i+1)\right\rceil$. Without loss of generality LET $C=\mathrm{Ts}^{d}$. Let $d, m>0$ be fixed. We infer $\mathrm{SWP}_{1}^{0}\left(\mathrm{Ts}_{m}^{d}\right)$ from $(\exists k>m) \mathrm{SWP}_{2}^{0}\left(\mathrm{Ts}_{k+1}^{d}, R_{k}\right)$, in $\mathrm{ACA}_{0}$. Suppose $k>\max \{d, m\}$ satisfies $\operatorname{SWP}_{2}^{0}\left(\operatorname{Ts}_{k+1}^{d}, R_{k}\right)$. Let $q:=k+1$. For any $n \geq 0$ let $a_{n}:=\#\left\{x \in \operatorname{Ts}_{k}^{d}:|x| \leq n\right\}=\left[z^{n}\right] G_{\mathrm{Ts}_{k}^{d}, \leq}(z)$ and $U_{k, i}:=\left\{x \in \operatorname{Ts}_{k}^{d}:|x| \leq|i|_{R_{k}}\right\}$. By the corresponding lower bound estimate (see 3.2 above) there exist $D>c_{k}>0$ such that $(\forall i \geq D)\left(\# S_{k, i}=a_{|i|_{R_{k}}}>c_{k} \cdot i\right)$. Choose sufficiently long sequence $y_{0}, y_{1}, \cdots, y_{M} \in \mathrm{Ts}_{m}^{d}, M>\max \{d, m, D\}$, such that $(\forall i \leq M)\left(\left|y_{i}\right| \leq K+i\right)$. Denote by $\oplus$ treeconcatenation in $\operatorname{Ts}^{d}$. To put it more exactly for any $t_{1}, t_{2} \in \mathrm{Ts}^{d}$ define $t_{1} \oplus t_{2} \in \mathrm{Ts}^{d}$ by substituting root of $t_{2}$ for the leftmost leaf of $t_{1}$. Denote by $\langle q\rangle^{d}$ a nested tuple $\left\langle\cdots\langle\underbrace{\cdots \cdots}_{q \text { times }} \overbrace{\cdots}^{d \text { times }} \in \mathrm{S}_{q}^{d}\right.$. Let $\langle q\rangle^{d} \in \operatorname{Ts}_{q}^{d}$ be a single-node tree with label $\langle q\rangle^{d}$ and let \langle\rangle$^{d} * l:=\underbrace{\left\langle\langle q\rangle^{d} \oplus \cdots \oplus\langle q\rangle^{d}\right.}_{l \text { times }} \in \operatorname{Ts}_{q}^{d}$. Let enum $_{U_{k, i}}: \# U_{k, i} \rightarrow U_{k, i}$ be strictly increasing (with respect to the underlying linearization $\leq_{C}$ ) enumeration of $U_{k, i}$. Let 
$x_{i}:=$

$$
\begin{cases}\langle q\rangle^{d} *(D+3-i) & \text { if } \quad i \leq D, \\ y_{|i|_{R_{k}}} \oplus\langle q\rangle^{d} \oplus \text { enum }_{U_{k, i}}\left(\# U_{k, i}-\left\lfloor c_{k} \cdot i\right\rfloor\right) & \text {,if } \quad D<i \leq M . \\ \oplus\langle q\rangle^{d} \oplus\left\langle\left\lfloor\left(c_{k}\right)^{-1}\left(1+\left\lfloor c_{k} \cdot i\right\rfloor\right)\right\rfloor-i\right\rangle^{d} & \end{cases}
$$

Let $K^{\prime}:=K+q(D+3)+\left\lfloor\left(c_{k}\right)^{-1}\right\rfloor$. Then for any $i \leq M$ we have $x_{i} \in \operatorname{Ts}_{q}^{d}=\operatorname{Ts}_{k+1}^{d}$ and $\left|x_{i}\right| \leq$ $K^{\prime}+2|i|_{R_{k}}$. Hence by the assumption there are $M$ and $i<j \leq M$ such that $x_{i} \unlhd x_{j}$. To complete the proof we show that it is only possible if $|i|<|j|, x_{i}=y_{|i|_{R_{k}}}$ and $x_{j}=y_{|j|_{R_{k}}}$. Consider all cases.

1. $x_{i}=\langle q\rangle^{d} *(D+3-i)$. So $x_{j}=\langle q\rangle^{d} *(D+3-j)$ is impossible, since $D+3-i>D+3-j$ implies $x_{j} \unlhd x_{i}$, but not $x_{i} \unlhd x_{j}$. But any other form of $x_{j}$ contains only two occurrences of $\langle q\rangle^{d}$, whereas $x_{j}$ at least three ones, and hence $x_{i} \unlhd x_{j}$ is not possible, either.

2. $x_{i}=y_{|i|_{R_{k}}} \oplus\langle q\rangle^{d} \oplus \operatorname{enum}_{U_{k, i}}\left(\# S_{k, i}-\left\lfloor c_{k} \cdot i\right\rfloor\right) \oplus\langle q\rangle^{d} \oplus\left\langle\left\lfloor\left(c_{k}\right)^{-1}\left(1+\left\lfloor c_{k} \cdot i\right\rfloor\right)\right\rfloor-i\right\rangle^{d}$,

where $i>D$, and hence $x_{j}=\langle q\rangle^{d} *(D+3-j)$ fails for $j>i>D$. Hence $x_{j}=y_{|j|_{R_{k}}} \oplus$ $\langle k\rangle^{d} \oplus \operatorname{enum}_{U_{k, i}}\left(\# U_{k, j}-\left\lfloor c_{k} \cdot j\right\rfloor\right) \oplus\langle q\rangle^{d} \oplus\left\langle\left\lfloor\left(c_{k}\right)^{-1}\left(1+\left\lfloor c_{k} \cdot j\right\rfloor\right)\right\rfloor-j\right\rangle^{d}$

where $j>i>D$. Consider subcases.

(a) Suppose $|i|_{R_{k}}=|j|_{R_{k}}$. Then $y_{|i|_{R_{k}}}=y_{|j|_{R_{k}}}$ and $U_{k, i}=U_{k, j}$.

i. Suppose $\left\lfloor c_{k} \cdot i\right\rfloor=\left\lfloor c_{k} \cdot j\right\rfloor$. Then $\left\lfloor\left(c_{k}\right)^{-1}\left(1+\left\lfloor c_{k} \cdot i\right\rfloor\right)\right\rfloor=$

$$
\begin{aligned}
& \left\lfloor\left(c_{k}\right)^{-1}\left(1+\left\lfloor c_{k} \cdot j\right\rfloor\right)\right\rfloor \text { and hence }\left\lfloor\left(c_{k}\right)^{-1}\left(1+\left\lfloor c_{k} \cdot i\right\rfloor\right)\right\rfloor-i> \\
& \left\lfloor\left(c_{k}\right)^{-1}\left(1+\left\lfloor c_{k} \cdot j\right\rfloor\right)\right\rfloor-j \text {, so }\left\langle\left\lfloor\left(c_{k}\right)^{-1}\left(1+\left\lfloor c_{k} \cdot j\right\rfloor\right)\right\rfloor-j\right\rangle^{d}<_{C} \\
& \left\langle\left\lfloor\left\lfloor\left(c_{k}\right)^{-1}\left(1+\left\lfloor c_{k} \cdot i\right\rfloor\right)\right\rfloor-i\right\rangle^{d} \text { and not }\left\langle\left\lfloor\left(c_{k}\right)^{-1}\left(1+\left\lfloor c_{k} \cdot i\right\rfloor\right)\right\rfloor-i\right\rangle^{d} \unlhd\right. \\
& \left\langle\left\lfloor\left(c_{k}\right)^{-1}\left(1+\left\lfloor c_{k} \cdot j\right\rfloor\right)\right\rfloor-j\right\rangle^{d} \text {, that implies } \neg\left(x_{i} \unlhd x_{j}\right) .
\end{aligned}
$$

ii. Suppose $\left\lfloor c_{k} \cdot i\right\rfloor<\left\lfloor c_{k} \cdot j\right\rfloor$. So \# $\# U_{k, i}-\left\lfloor c_{k} \cdot i\right\rfloor>\# U_{k, j}-\left\lfloor c_{k} \cdot j\right\rfloor$, hence enum $_{U_{k, i}}\left(\# U_{k, j}-\left\lfloor c_{k} \cdot j\right\rfloor\right)<_{C}$ enum $_{U_{k, i}}\left(\# U_{k, i}-\left\lfloor c_{k} \cdot i\right\rfloor\right)$, that by the same token implies $\neg\left(x_{i} \unlhd x_{j}\right)$.

(b) Suppose $|i|_{R_{k}}<|j|_{R_{k}}$. Then $y_{|i|_{R_{k}}} \unlhd y_{|j|_{R_{k}}}$ must be the case, as desired. 


\section{Acknowledgements}

We are grateful to the John Templeton Foundation and the Nederlandse Organisatie voor Wetenschappelijk Onderzoek (B 60-62). We are also grateful to Jan Christoph Schlage-Puchta and the AofA'10 referees for useful comments and suggestions.

\section{References}

[1] T. Arai, On the slowly well orderedness of $\varepsilon_{0}$, Math. Log. Q. 48(1) (2002) 125-130

[2] A. Bovykin and A. Weiermann: Unprovability, phase transitions and the Riemann zeta function. To appear in the proceedings of: New directions in the theory of universal Zeta- and L-functions

[3] W. Buchholz, E. A. Cichon, A.Weiermann, A uniform approach to fundamental sequences and hierarchies, Math. Log. Q. 40(2) (1994) 273-286

[4] Ph. Flajolet, R. Sedgewick, Analytic Combinatorics, Cambridge University Press, 2009

[5] H. Friedman, Some systems of second order arithmetic and their use, Proc. of the International Congress of Mathematicians (Vancouver, B. C., 1974), Vol. 1, Canad. Math. Congress, Montreal, Que. (1975) 235-242

[6] H. Friedman, Systems of second order arithmetic with restricted induction, I, II, Meeting of the Association for Symbolic Logic, Journ. of Symb. Log. 41(2) (1976) 557-559

[7] L. Gordeev, Generalizations of the one-dimensional version of the Kruskal-Friedman theorems, Journ. of Symb. Log. 54 (1987) 100-121

[8] L. Gordeev, Generalizations of the Kruskal-Friedman theorems, Journ. of Symb. Log. 55 (1990) $157-181$

[9] L. Gordeev, Quasi-Ordinals and proof theory, Proc. Conference on Graph Minors, Contemporary Mathematics 147 (1993) 485-494

[10] L. Gordeev, A modified sentence unprovable in PA, Journ. of Symb. Log. 59 (1994) 1154-1157

[11] L. Kirby, J. Paris, Accessible independence results for Peano Arithmetic, Bull. Lon. Math. Soc. 14 (1982) 285-293

[12] I. Kriz, Well-quasiordering finite trees with gap-condition. Proof of Harvey Friedman's conjecture, Annals of Mathematics 130 (1989) 215-226

[13] A. Montalbán, Computable linearizations of well-partial-orderings, Order 24(1) (2007) 39-48

[14] J. Paris, L. Harrington, A mathematical incompleteness in Peano arithmetic, in: J. Barwise (Ed.), Handbook of Mathematical Logic, North-Holland, Amsterdam, 1977

[15] S. G. Simpson, Subsystems of second order arithmetic, Perspectives in Logic (2nd ed.), Cambridge University Press, 2009 
[16] R. Smith, The consistency strength of some finite forms of the Higman and Kruskal theorems, in: Harvey Friedman's Research on the Foundations of Mathematics, North-Holland, Amsterdam (1985) 119-136

[17] A. Weiermann, An application of graphical enumeration to PA, J. Symbolic Logic 68 (2003) 5-16

[18] A. Weiermann, A classification of rapidly growing Ramsey functions, Proc. Amer. Math. Soc. 132 (2004) 553-561

[19] A. Weiermann, Classifying the provably total functions of PA, Bull. Symbolic Logic 12(2) (2006) $177-190$

[20] A. Weiermann, Phase transition thresholds for some Friedman-style independence results, Math. Logic Q. 53(1) (2007) 4-18

[21] A. Weiermann, Phase transitions for Gödel incompleteness, Ann. Pure Appl. Logic 157 (2-3) (2009) 281-296. 